\title{
Antenatal Screening for Syphilis: Ten Years of Observation Amongst Pregnant Women Receiving Care at the Maternity Units of the Province of Trento in Northern Italy
}

\author{
Silvano Piffer ${ }^{1, ~}$, Mariangela Pedron ${ }^{1}$, Anna Lina Lauriola ${ }^{2}$, Roberto Rizzello ${ }^{1}$ \\ ${ }^{1}$ Clinical and Evaluational Epidemiology Service, Provincial Health Agency, Trento, Italy \\ ${ }^{2}$ Paediatrics Unit, S. Maria Del Carmine Hospital, Rovereto, Provincial Health Agency, Trento, Italy
}

Email address:

piff.silv.tab@gmail.com (S. Piffer)

${ }^{*}$ Corresponding author

To cite this article:

Silvano Piffer, Mariangela Pedron, Anna Lina Lauriola, Roberto Rizzello. Antenatal Screening for Syphilis: Ten Years of Observation Amongst Pregnant Women Receiving Care at the Maternity Units of the Province of Trento in Northern Italy. Journal of Gynecology and Obstetrics. Vol. 9, No. 3, 2021, pp. 59-65. doi: 10.11648/j.jgo.20210903.12

Received: April 26, 2021; Accepted: May 12, 2021; Published: May 21, 2021

\begin{abstract}
Introduction. Serology screening for syphilis infection in pregnancy is an internationally recommended practice. For some time, in the province of Trento (northern Italy), data regarding antenatal serology screening for syphilis have been acquired through the birth assistance certificate, whose completion by the healthcare professionals providing assistance at birth is mandatory in Italy. This study describes the coverage of serology screening and the seroprevalence of syphilis infection amongst pregnant women receiving care in the maternity facilities of the province of Trento between 2008 and 2017. Materials and methods. The results of serological screening are recorded in the electronic birth assistance certificate as: test not performed; negative test, positive test, or results pending. In cases of a positive test or results pending, the Authors consulted the hospital information system (SIO), an electronic repository containing data regarding all services provided to users of the Provincial Health Service. This operation also made it possible to recover full data regarding the serological profile, any treatments administered and the obstetric/neonatal outcomes. The Authors calculated screening coverage, the seroprevalence of the infection and the occurrence of cases of congenital syphilis, by analysing the neonates' health status for the first two years of life. Results. Between 01.01.2008 and 31.12.2017, 46,627 pregnant women received care at maternity units of the province of Trento. Screening coverage was found to be higher than $99 \%$. A total of 95 pregnant women were found to be true positives at serology screening for syphilis (15 Italian nationals and 80 foreign women). Seroprevalence for all pregnant women was 2/1000 (0.4/1000 amongst Italian women and 6.8/1000 amongst foreign women). Amongst the latter, the highest value was observed amongst women originating from Eastern European countries (9.3/1000). 62\% of positive cases were identified as prior, already treated syphilis and 38\% were identified as an early or late latent infection. Antibiotic therapy with penicillin was prescribed in all cases for which it was indicated. No considerable differences were observed with regard to the obstetric/neonatal outcomes between seropositive ad seronegative women. No cases of congenital syphilis were observed. Conclusions. Screening coverage concerned almost all the pregnant women who received care. The seroprevalence calculated in this study was consistent with the available Italian and European data. Our findings confirmed that there is a higher proportion of cases amongst the foreign population, especially amongst women from Eastern European countries, who nevertheless have an antenatal care profile not unlike that of seronegative women.
\end{abstract}

Keywords: Syphilis, Pregnancy, Congenital Syphilis, Obstetric/Neonatal Outcomes, Current Information Flows

\section{Introduction}

Syphilis is a prevalently sexually-transmitted infectious disease caused by the spirochete bacterium Treponema
Pallidum. In recent decades, the disease has followed an incremental trend, especially in developing and Eastern European Countries, with a consequent impact also on more developed countries, due to migration $[1,2]$. Changes in the 
sexual habits of the younger population have further contributed to the re-emergence of the disease [3]. In 2016, the WHO estimated that there were 63 million prevalent cases of syphilis, of which approximately $90 \%$ in developing countries [4]. It should also be pointed out that there is frequently concomitance with HIV positivity, which worsens the evolution of the disease [5]. In Italy, two systems are used to monitor sexually-transmitted diseases (STDs), including Syphilis, one based on clinical facilities, the other on clinical microbiology lab data, and both coordinated by the National Institute of Health. These surveillance systems indicate that between 1991 and 2015, there was a considerable increase in reported cases of primary and secondary syphilis, as well as latent syphilis. At the same time, an increase was detected in the prevalence of HIV positivity amongst the cases of STDs and an increase in the prevalence in foreign nationals, which, in 2015, had reached $9.9 \%$ and $19 \%$, respectively [6]. A syphilis infection contracted during pregnancy that goes untreated can result in serious foetal and neonatal health problems, given the possibility of vertical maternal-foetal transmission. Every year, worldwide there are approximately 12 million new cases of syphilis, of which two million in pregnant women $[7,8]$. The WHO estimates that maternal luetic infection is responsible for between 715,000 and $1,575,000$ cases of congenital syphilis, foetal or perinatal death and low birth weight [8] with considerable consequences on the product of conception: 460,000 miscarriages or intrauterine deaths, 270,000 cases of congenital syphilis and 270,000 premature neonates or with a low birth weight [9]. During pregnancy, infection can not only be prevented, but also detected early, by means of serology screening, which is usually performed in the first trimester but that, in the case of high-risk populations or those with a high prevalence of the disease, is repeated at the 28th week of pregnancy and at the time of the birth $[10,11]$. As in many other countries, in Italy serology screening for syphilis is one of the recommended antenatal monitoring practices and is therefore provided to pregnant women free of charge, without any co-payment [12]. In the province of Trento, the results of serology screening for syphilis infection have been recorded in the Birth Assistance Certificate (BAC) for some time. Throughout Italy, the completion of this document by the healthcare professionals present at the time of the birth is mandatory by law [13]. This paper provides data on the seroprevalence of syphilis infection in the pregnant women who delivered at the maternity units of the province of Trento between 2008 and 2017, and analyses the obstetric and neonatal outcomes.

\section{Materials and Methods}

The BAC used in the province of Trento acquires more pregnancy-related parameters than is required by the ministerial template. More specifically, it also acquires information on antenatal serology screening for various infections and, in this particular case that for syphilis. The operating protocol for antenatal serological syphilis screening in the province of Trento is in accordance with the guidance issued by a national multidisciplinary group [14]. More specifically, the protocol consists initially in the performance of not anti-treponemal tests such as the VDRL (Venereal Disease Research Laboratory Test) and the RPR (Rapid Plasma Reagin test) and, in the case of a positive result on these tests, the performance of a confirmation treponemal test, usually the TPHA (Treponema Pallidum haemagglutination assay) or TPPA (Treponema Pallidum Particle Agglutination assay). In the presence of an ambiguous result, a specific IgM and IgG antibody immunoblot assay is performed. The results of the serology testing for syphilis are recorded in the pregnant woman's personal antenatal guide and are later recorded in the electronic BAC database at all the maternity units of the province of Trento, by the midwives present at the time of the birth. The criteria for the recording of serology screening are: test not performed, negative test, positive test, results pending. For cases recorded in the $\mathrm{BAC}$ as results pending or positive test, we consulted and retrieved data from the hospital information system (SIO), a repository used to store all the clinical information regarding both residents and non-residents who receive any service from the Provincial Health Service. The consultation of the hospital information system made it possible to ascertain the true positives and to proceed, by retrieving the entire battery of tests, with their clinical classification. The hospital information system was also used to analyse the use of antibiotic therapy amongst pregnant women with a positive test and an indication for the treatment, and to ascertain the distant outcomes in neonates with seropositive mothers. The coverage of serological screening over all pregnant women who gave birth in hospital maternity units of the province of Trento between 2008 and 2017 was analysed. We analysed the coverage trend over time, also in relation to the age group, marital status, academic qualifications and nationality of the pregnant women, as well as in relation to the maternity facility. We calculated the seroprevalence of syphilis infection, and its trend over time and in relation to the woman's age group, marital status, academic qualification and nationality. Trend significance was analysed using the Cochran-Armitage criterion and the significance of the differences between the proportions in the various subpopulations compared was analysed using the chi squared test or Fisher's exact test. The prevalence values obtained were reported together with the 95\% confidence intervals. The obstetric outcomes and the neonatal outcomes for positive mothers were analysed by comparing them with those of negative mothers. The neonates' health was assessed at birth and up to the age of two years.

\section{Results}

Between 01.01.2008 and 31.12.2017, 46,627 pregnant women received care at maternity units of the province of Trento. The corresponding number of live births for the same period was 47,759 . The average age of the pregnant women rose from 31.9 years in 2008 to 32.3 years in 2017 and the percentage of those of foreign nationality increased from 
$21.7 \%$ in 2008 to $26.20 \%$ in 2017 . The coverage of serology screening for syphilis remains, for each year, above $99 \%$, without significant variations from one year to the next. As a matter of fact, coverage improved over time, so that the percentages of untested pregnant women dropped from $0.8 \%$ in 2008 to $0.2 \%$ in 2017 . Screening coverage did not vary in relation to the age group, marital status, academic qualification or nationality of the mother or in relation to the maternity facility. During the study period, 138 women were recorded in the BAC as having a positive serology test for syphilis, with a mean positive mother rate of $2.95 \%$ and with an annual range of between 1.61 and $4.04 \%$ (figure 1). The consultation of the hospital information system also made it possible to ascertain that of the 138 cases registered in the BAC, only 95 (69\%) were true positive cases, of which 15 Italian women and 80 foreign nationals, with an average of 9.5 cases per year. The distribution of true positive cases for each calendar year is shown in figure 2 . The cases incorrectly recorded as positive in the BAC actually regarded: 1 untested case, 3 false positives and 39 negative cases. As regards the number of true confirmed positive cases, the average value of antenatal syphilis seroprevalence, over the entire study period, was equal to $2.03 / 1.000(95 \% \mathrm{CI}$ : $1.92-2.14)$ with a trend per single year as shown in figure 3 . In the years 2015 2016, there was an increase in seroprevalence; however, this value was not statistically significant in relation to the average for the period and was associated more with a decrease in the number of pregnant women and consequently in births than with an increase in positive cases. Seroprevalence was seen to be higher amongst separated/divorced women $(7.7 \%$, 95\% CI: 6.3-9.0) and amongst pregnant women whose academic qualification was lower secondary school certificate or lower $(8.8 \%$, $95 \% \mathrm{CI}$ : 7.0-10.5); it did not vary significantly in relation to the age group of the pregnant woman, although it was seen to be higher in the 25-29 years and 35-39 years brackets (table 1). Seroprevalence varies considerably in relation to nationality, where Italian women have a value of $0.42 / 1.000$ (95\% CI: $0.39-0.45)$ and foreign women a value of $6.87 / 1.000(95 \%$ CI: 6.64-7-10). The difference between foreign and Italian women is statistically significant $(\mathrm{p}<0.0001)$. Amongst foreign women seroprevalence was seen to be higher amongst those coming from Eastern European countries and Africa (figure 4). Amongst the 95 positive cases, there was also co-positivity for HBsAg (4 cases), for HCV (6 cases) and for HIV ( 2 cases). The co-positivity regarded Italian women in two cases and foreign women in 10 cases. The demographic and obstetric characteristics of the infected women, compared to those not infected, receiving care in the maternity facilities of the province during the study period are provided in table 2. Amongst infected mothers, compared to those not infected, statistically significant excesses were observed in relation to the proportion of separated/divorced women $(p<0.001)$, the proportion of foreign women $(p<0.00001)$, the lower proportion of graduates $(p<0.001)$, and the higher proportion of subjects with low academic qualifications $(p<0.05)$. The clinical characteristics of the positive mothers are shown in table $3.62 \%$ of cases regarded prior, already treated infection with a serological scar outcome and $37.8 \%$ of cases were identified as early or late latent infection. The neonatal outcomes observed amongst the 97 live births of the 95 seropositive pregnant women (2 twin births) and the 47,759 live births during the study period are shown in table 4. Overall, excesses were seen amongst the babies of the seropositive mothers, although the only statistically significant excess regards the proportion of neonates hospitalised at birth $(\mathrm{p}<0.05)$. Just two neonates were found to be symptomatic at birth (lymphadenopathy and hepatosplenomegaly, retarded growth). The retrieval of the clinical and serological data up to the age of two years using the hospital information system for 82 of the 96 live births did not make it possible to identify any case of congenital syphilis.

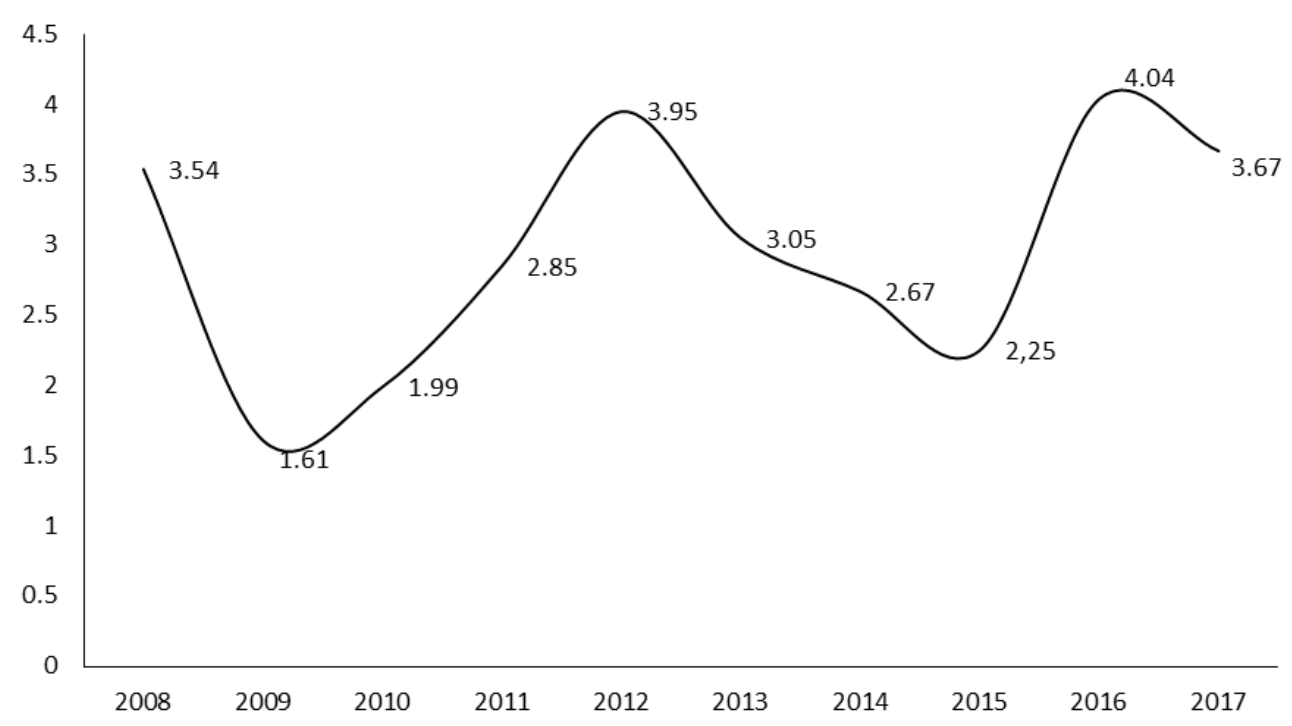

Figure 1. Province of Trento. Pregnant women recorded in the birth assistance certificate as testing positive at the serological screening for syphilis. Proportion/1,000. Per single year. Period 2008-2017. 


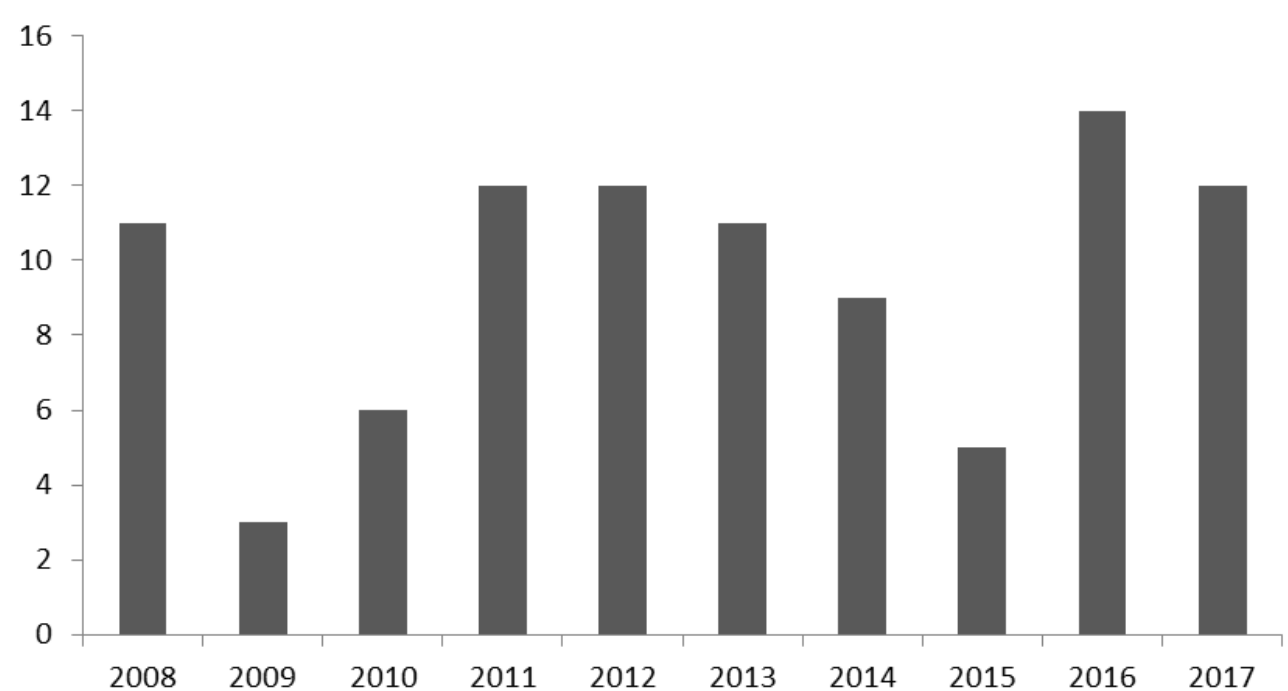

Figure 2. Province of Trento. Number of women who actually tested positive at the serological screening for syphilis. Per single year. Period $2008-2017$.

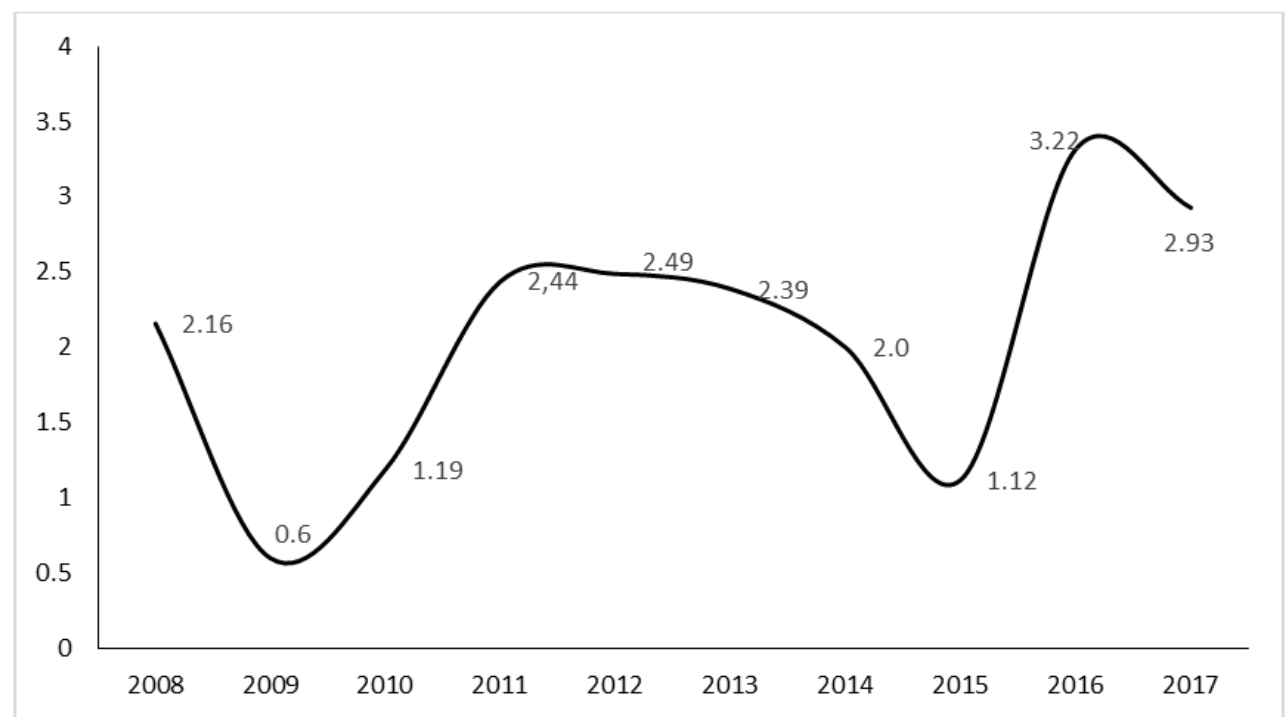

Figure 3. Province of Trento. Seroprevalence of syphilis infection/1,000 during pregnancy. Per single year. Period 2008-2017.

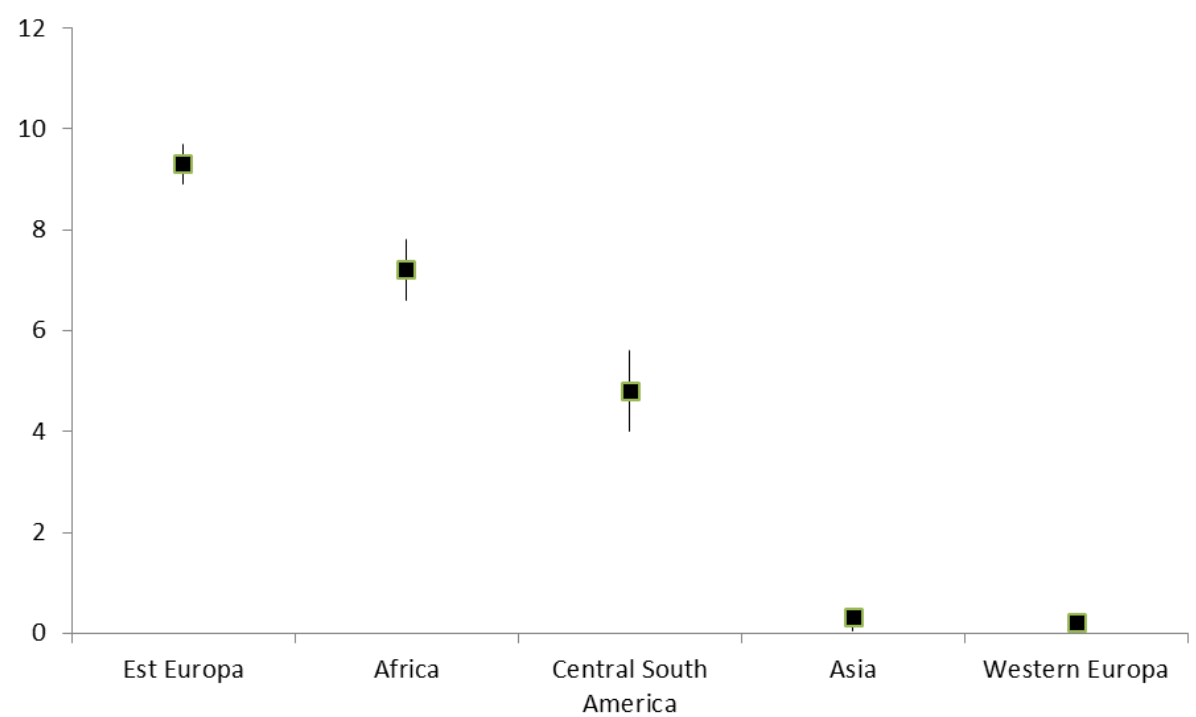

Figure 4. Province of Trento. Seroprevalence and 95\% CI of syphilis infection during pregnancy in mothers of foreign origin. By geographical area of origin. Period: 2008-2017. 
Table 1. Province of Trento. Seroprevalence of syphilis infection during pregnancy. By age group. Period 2008-2017.

\begin{tabular}{lll}
\hline Age group & Seroprevalence / 1,000 & $\mathbf{9 5 \%} \mathbf{C I}$ \\
\hline$<=24$ years & 1.90 & $1.69-2.29$ \\
$25-29$ & 2.34 & $2.10-2.49$ \\
$30-34$ & 1.76 & $1.58-1.94$ \\
$35-39$ & 2.26 & $2.10-2.44$ \\
$40+$ & 1.78 & $1.63-1.93$ \\
\hline
\end{tabular}

Table 2. Province of Trento. Demographic and obstetric characteristics of mothers with syphilis infection compared to those with negative test. Period 20082017.

\begin{tabular}{lll}
\hline Parameter & Positive at serological screening & Negative at serological screening \\
\hline Average age and range (years) & $30(22-43)$ & $32(15-45)$ \\
\% unmarried & 31.6 & 28.5 \\
\% separated/divorced & 11.5 & 3.1 \\
\% foreign nationals & 52.6 & 25.4 \\
\% of graduates & 11.6 & 31.9 \\
\% with lower secondary school certificate or lower academic qualifications & 9.4 & 2.2 \\
\% primiparous & 44.0 & 45.2 \\
\% first antenatal appointment by 13 weeks & 86.1 & 73.5 \\
\% caesarean section (elective, emergency) & $28.0 \%$ & 25.3 \\
\hline
\end{tabular}

Table 3. Province of Trento. Clinical characteristics of pregnant mothers with syphilis infection. receiving care in the maternity units of the province of Trento in the period 2008-2017.

\begin{tabular}{ll}
\hline Clinical condition & Frequency \\
\hline Prior infection, treated in the past (serological scar) & 45 \\
Early latent infection, started treatment during the current pregnancy & 22 \\
Prior infection, not known whether treated previously & 14 \\
Late latent infection, started treatment during the current pregnancy & 12 \\
Late latent infection, not known whether treated previously & 2 \\
Total & 95 \\
\hline
\end{tabular}

Table 4. Province of Trento. Neonatal outcomes amongst pregnant women with and without syphilis infection Period 2008-2017.

\begin{tabular}{lll}
\hline Parameter & Mothers positive at serological screening & Mothers negative at serological screening \\
\hline Still births & $10.3 / 1000$ & $2.9 / 1000$ \\
Apgar $<8$ & 5.1 & 2.4 \\
Pre-term & 7.2 & 6.9 \\
Low birth weight & 7.2 & 7.0 \\
Stunted intra-uterine growth & 1.6 & 1.3 \\
Hospitalised at birth & 11.3 & 6.3 \\
Breast-fed only & 85.2 & 8.3 \\
\hline
\end{tabular}

\section{Conclusions}

Antenatal serology screening for syphilis is justified by the considerable neonatal morbidity and mortality associated with congenital syphilis and their potential predictability [13]. This screening has a favourable cost-benefit ratio, even when the prevalence of the disease amongst pregnant women is just $0.005 \%[7,9,11]$. Early identification of the infection and adequate antibiotic treatment with penicillin practically offsets the risk of congenital syphilis in babies born to seropositive mothers $[15,16]$. The possibility of acquiring data regarding antenatal screening for syphilis through a current information flow such as the BAC makes it possible to perform analyses regarding coverage and seroprevalence and therefore its transmissibility to the foetus in potentially all the pregnant women receiving care in a given area. This also makes easy to carry out follow-up assessments on newborns. In the province of Trento, the coverage of serological screening was found to be very high, more than $99 \%$, without any differences in relation to the characteristics of the pregnant women and the maternity facilities attended. The highest value, $99.7 \%$, was achieved in 2017 . Every effort should be made to guarantee $100 \%$ cover, considering that each mother-child pair should not leave the maternity facility without having been tested $[10,11]$. As a matter of fact, the data acquired directly from the BAC do not make it possible to perform a very accurate estimate of seroprevalence, considering the existence of a certain degree of data entry error that tends to overestimate it. In the province of Trento, the availability of a tool such as the SIO makes it possible to optimally estimate seroprevalence, especially in the early 
years of the study, in which there were the greatest gaps in data entry. Some of the data entry errors should be attributed to the difficulties encountered by the healthcare professionals who are usually involved in the antenatal care, in particular midwives, in correctly interpreting the serology of the disease, which is still difficult and requires consolidated experience [14]. The seroprevalence of syphilis infection during pregnancy observed in our retrospective study would appear to be consistent with previous Italian and European studies $[17,18]$. The province of Trento, like the rest of Italy, was confirmed as being an area in which endemic syphilis is low. The study also confirmed a certain association with the personal characteristics of the pregnant women that can be correlated with potentially higher-risk lifestyles and behaviours, aspects that are also not routinely recorded in either the BAC or the SIO. However, the cases of coinfection with HIV would appear to far more limited than reported in literature $[5,7,8]$. No statistically significant differences were observed in relation to age group. As reported previously, seroprevalence was seen to be far higher amongst foreign women, especially those coming from Eastern European countries and Africa [1-4, 18-21]. On a clinical level, in most cases it regarded prior forms or early or late latent forms that had already been treated, not always adequately and for the appropriate period, especially in the case of foreign women who acquired the infection in their country of origin. In the province of Trento, foreign women from Eastern Europe and Africa represent a sub-population that deserves special attention and for whom global health promotion programmes should be developed [22-24]. However, it should be noted that these categories of women were not seen to receive less antenatal care, as shown by the number of antenatal appointments and their distribution over the course of the trimesters. Well-organised hospital and community antenatal care may certainly help reduce or mitigate the impact of social inequalities on health [7-9, 17]. This could also explain why no considerable differences were observed in obstetric-neonatal indicators amongst seropositive mothers compared to seronegative women, even when we exclude the subjects for whom it is not possible to find follow-up documentation, a large proportion of which were born to foreign mothers. We are confident that similar experiences can develop in other Italian regions in order to have a more consistent case history.

\section{References}

[1] Cohen SE, Klausner JD, Engelman J et al. Syphillis in the modern era: an update for physicians. Infect Dis Clin North Am 2013 Dec. 27 (4): 705-720.

[2] World Health Organization. Progress report of the implementation of the global strategy for prevention and control of sexually transmitted infections: 2006-2015. Geneva: WHO; 2015.

[3] WHO. Syphillis screening and treatment for pregnant women. WHO Geneve 2017 Available from http://apss.who.int/inis.
Last Accessed January 92021.

[4] Korenromp EL, Rowley J, Alonso M, Mello MB, Wijesooriya NS, et al. (2019) Global burden of maternal and congenital syphilis and associated adverse birth outcomes-Estimates for 2016 and progress since 2012. PLOS ONE 14 (2): e0211720. Available from https://doi.org/10.1371/journal.pone.0211720. Last accessed January 102021.

[5] Sexton J, Garnett G, Rettingen JA. Metaanalysis and metaregression in interprewting study variability in the impact of sexually transmitted diseases on susceptibility to HIV infection. Sex Transm Dis 2005, 32 (6): 351-357.

[6] Salfa MC, Ferri M, Suligoi B e la rete sentinella dei centri clinici e dei laboratori di microbiologia clinica per le infezioni sessualmente trasmesse: aggiornamento dei dati dei due sistemi di sorveglianza sentinella attivi in italia al 31 dicembre 2015. Not Ist Super Sanità 2017; 30 (7-8): 3-27.

[7] Walker DG, Walker GJA. Prevention of congenital syphilis-time for action. Bullettin of World Health Organization. 2004; 82 (6).

[8] Simms I, Broutet N. Congenital Syphilis re-emerging. JDDG 2008; 6: 269-272.

[9] Saloojee H, Velaphi S et al. The prevention and management of congenital syphilis: an overview and recommendations. Bullettin of the World Health Organization. 2004; 82: 424-430

[10] Centers for Disease and Control and Prevention. Sexually transmitted diseases treatment guidelines 2010. MMWR 2010; 59 (RR12): 1-110.

[11] United States Preventive Services Task Force. Screening for syphilis infection: recommendation statement. Rockville (MD): Agency for Healthcare Research and Quality (AHRQ); JAMA 2018: 320 (9) 911-917.

[12] Ministero della Salute, Istituto Superiore di Sanità e CeVEAS. Available from http://www.salute.gov.it/imgs/C_17_pubblicazioni_1436_alle gato.pdf. Last Accessed January 52021.

[13] Ministero della Salute. Il Certificato di Assistenza al Parto. DM 16 luglio 2001, n. 349.

[14] Gruppo multidisciplinare "Malattie infettive in ostetriciaginecologia e neonatologia" (AMCLI, SIMaST, SIMIT, SIN, SIP). Percorsi diagnostico-assistenziali in Ostetricia e Neonatologia. Sifilide; Aprile 2012.

[15] Delport SD, Pattinson RC. Syphilis: prevention, diagnosis and management during pregnancy and infancy. In Newell ML and McIntyre eds: Congenital and perinatal infections. Cambridge University Press, UK 2000; 13: 258-75.

[16] Watson-Jones D, Gumodoka B et al. Syphilis in Tanzania. The effectiveness of antenatal syphilis screening and single dose benzathine penicillin treatment for the prevention of adverse pregnancy outcomes. J Infect Dis 2002; 186: 948-957.

[17] Genç M, Ledger W. Syphilis in pregnancy. Sex Transm Infect 2000; 76: 73-79.

[18] Tridapalli E, Capretti MG et al. Congenital syphilis in Italy: a multicentre study. Arch Dis Child Fetal Neonatal 2010; Sep 24.

[19] Zammarchi L, Borchi B, Chiappini E et al. Syphilis in pregnancy in Tuscany, description of a case series from a global health perspective. J. Mat-Fet \& Neonat. Med 2012; 25 (12): 2601-2605. 
[20] Roncorati G, Fazio C, Gaspari V et al. Syphilis in a highdensity urban area in the north of Italy. New Microbiologica; 2019; 42 (3): 166-170.

[21] Marangoni A, Moroni A, Tridapalli E, et al. Antenatal syphilis serology in pregnant women and follow-up of their infants in northern Italy. Clin Microbiol Infect 2008 (14): 1065-1068.

[22] Workowski KA, Berman SM. Sexually transmitted diseases treatment guidelines. MMWR Recomm Rep. 2006; (4); 55: 1-94.
[23] Suligoi B, Giuliani M. Sexually transmitted disease among foreigners in Italy. Migration Medicine Study Group. Epidemiol. Infect 1997; 118 (3): 235-241.

[24] Giuliani M, Suligoi B. Differences between not national and indigenous patients with sexually transmitted infections in Italy and insight into the control of sexually transmitted infections. Sex transm Dis 2004; 31 (2): 79-84. 